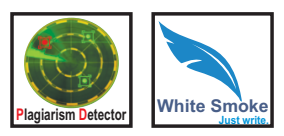

\title{
Identification of promising sources for drought tolerance in cultivated and wild species germplasm of barley based on root architecture
}

\begin{tabular}{l} 
Paper received: 03.09.2018 \\
\hline Authors Info \\
Manju', V. Kaur"*, K. Sharma ${ }^{1}$ \\
and A. Kumar' \\
'Department of Botany, Baba \\
Mastnath University, \\
Rohtak- 124 021, India \\
'Division of Germplasm Evaluation, \\
ICAR- National Bureau of Plant \\
Genetic Resources, \\
New Delhi-110 012, India \\
\hline vorresponding Author Email : \\
vikender.kaur@icar.gov.in
\end{tabular}

\section{Edited by}

Dr. R.B. Raizada

Reviewed by

Dr. Suritha Rajkumar

Dr. Anita K.
Abstract

Aim : Root architecture of 220 diverse barley germplasm of Indian (134) and exotic (86) origin was evaluated for polyethylene-glycol simulated drought stress to identify drought tolerant genotypes.

Methodology : The evaluation of root images was done using root scanner (WinRHIZO Pro software v2009). Variance, Principal Component Analysis (PCA) and Ward's agglomerative hierarchical clustering were carried out using SAS software. Correlation matrices were generated using $R$.

Results : Analysis of variance indicated that under stress treatment, differences among the tested germplasm accessions were highly significant $\left(\mathrm{P}_{0.01}\right)$ with respect to total root length $(\mathrm{RL})$, seminal root number (SRN), root surface area (RSA), root volume (RV), root diameter (RD), lateral root number (LRN) and root dry weight (RDW) per seedling. LRN was stimulated while other root traits such as RL, RSA, RV and RDW were significantly inhibited under stress. PCA indicated that first three components accounted for $80.50 \%$ of the total multivariate variation with $\mathrm{PC} 1$ accounting for $44.83 \%$, PC2 for $19.84 \%$ and PC3 for $15.87 \%$ and it was mainly explained by RL, RSA, RV and RDW. Cluster analysis grouped 220 barley accessions into five major clusters, with cluster I being drought

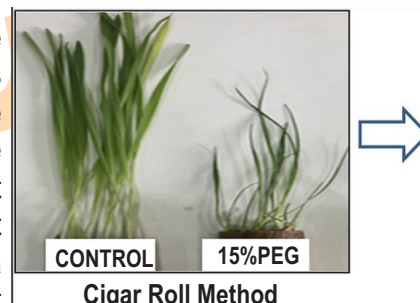

Cigar Roll Method
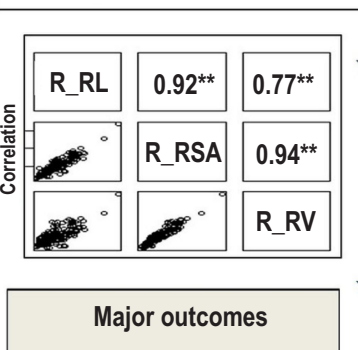
$>$ Drought tolerant genotypes identified $>$ Divergent accession groups identified

$>$ Correlation among root traits

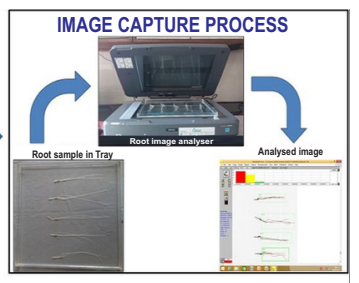

Root Phenotyping

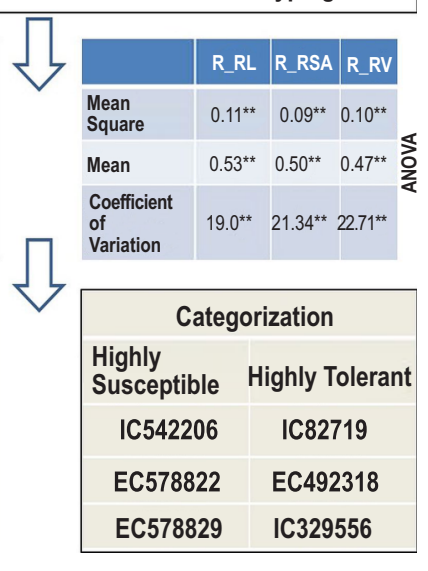
susceptible, cluster II being drought tolerant, cluster III being moderately drought tolerant, Cluster IV being highly drought tolerant and Cluster $V$ being highly drought sensitive, respectively.

Interpretation : Accessions IC393980, IC082719, IC329556, EC492318, EC578789, EC578790, IC335811 and wild barley $H$. marinum ssp. gussoneanum proved to be potential genetic resources for drought tolerance, which can be used in cereal breeding program for rain-fed agriculture.

Key words: Barley germplasm, Drought stress, Root architecture, Root traits, Wild species

How to cite : Manju, V. Kaur, K. Sharma and A. Kumar: Identification of promising sources for drought tolerance in cultivated and wild species germplasm of barley based on root architecture. J. Environ. Biol., 40, 309-315(2019). 


\section{Introduction}

Barley (Hordeum vulgare L.) is a multipurpose crop, ranked fourth among the worldwide production of cereals (FAO, 2017; Druka et al., 2011). It is an important source of feed and forage for livestock in an agriculture dominant country like India, particularly during fodder scarcity months of winter season in dryland/rain-fed areas. Besides its traditional use for food and feed, it is gaining popularity as a commercial crop under contract farming system to assure supply for brewing and malting industries. Although it is an ancient crop in India, its cultivation is presently facing stiff challenges in terms of area with wheat in winter season and less remunerative prices since post green revolution era. Further, about $44 \%$ area under barley cultivation in India is rain-fed and more than $60 \%$ area under cultivation in Rajasthan- the major barley producing state, is unirrigated. Owing to the growing conditions of barley in marginal areas on residual moisture, these areas lack optimum productivity (Ceccarelli et al., 1999; Kumar et al., 2013).

The best option to improve crop yield under drought stress is to develop drought-tolerant crop varieties (Ashraf et al., 2009; Pandey et al., 2010; Kaur et al., 2016, 2017). The germplasm accessions including landraces and wild relatives of barley are reservoirs of a range of allelic variants, which can be used in breeding especially for tolerance to abiotic stresses such as drought, heat, salinity, as well as pathogens (Shavrukov et al., 2010; Uçarlı et al., 2016). Breeder selection and farmers preference have led to narrow genetic base of barley with reduced allelic diversity to tolerate abiotic/biotic stresses. The problem of genetic uniformity in modern cultivars can be circumvented by using diverse germplasm in the breeding programs. In recent years, identification of trait specific accessions and discovery of novel alleles for different traits from germplasm collections for use in breeding programs have emerged as important approach for crop breeding under climate change (Kumar et al., 2010; Kaur et al., 2018).

The root system architecture (RSA) of a crop can influence the efficiency of water capture and extraction, and thus may serve as drought avoidance mechanism (Pennisi, 2008). An advantage of having superior root traits such as seminal root number, branching, root diameter, total root length/surface area, root length density, root volume and root hairs under drought stress conditions have been proved recently in rice, wheat and maize (Gowda et al., 2011; Henry et al., 2012; Christopher et al., 2013; Uga et al., 2013). While a number of studies have examined above ground water-use traits in barley (Diab et al., 2004; Teulat et al., 2001, 2003; Chen et al., 2010), scanty information is available on the extent of genetic variation in root morphological traits in response to drought stress in genebank germplasm collections. Therefore, the present study was conducted to evaluate root architecture variability in a diverse collection of barley under polyethylene glycol (PEG) induced osmotic stress as a profound base for improving drought tolerance.

\section{Materials and Methods}

Plant material: A total of 220 germplasm of spring barley, out of which 134 are indigenous and 86 exotic collections, were procured procured from National Gene Bank of ICAR-National Bureau of Plant Genetic Resources (NBPGR), New Delhi, India. The diverse accessions used in the present study were selected from a large collection of 5000 barley germplasm accessions subjected to diversity analysis, based on agro-morphological and phenological traits using PowerCore (v.1.0) software.

Germination paper rolls culture conditions for root traits: To impose PEG induced drought stress, first the seeds were surface sterilized with $2 \%$ Bavistin (w/v for $10 \mathrm{~min}$ ) followed by sodium hypochlorite $2 \%$ (v/v for $10 \mathrm{~min}$ ) using horizontal shaker and then rinsed five times with sterilized distilled water. The sterilized seeds were immersed in distilled water for another $8 \mathrm{hr}$ at $20^{\circ} \mathrm{C}$ in dark and then germinated in aseptic petri dishes (diameter $90 \mathrm{~mm}$ and height $15 \mathrm{~mm}$ ) paved with damp germination paper. After sprouting for 3 days at room temperature, one set of the seedlings was subjected to stress treatment viz. 15\% PEG-6000 (-0.295 $\Psi_{\mathrm{S}}$ at $25^{\circ} \mathrm{C}$ ) for a period of 10 days in a growth chamber under longday photoperiod (16 hr light $/ 8 \mathrm{hr}$ dark) at $22^{\circ} \mathrm{C}$ and a relative humidity of $65-75 \%$ using germination paper roll method (Zhu et al., 2005). Another set was kept as control in half strength Hoagland solution (Hoagland and Arnon, 1950) under similar growth conditions as stress treatment set. On $10^{\text {th }}$ day, the seedlings were preserved in $30 \%$ ethanol to prevent further growth. Consequently, all measurements pertaining to root phenotyping were taken at same time point.

Root phenotyping: The roots were cut from root-shoot junction, and were spread out in a root positioning tray $(30 \times 40 \mathrm{~cm})$ with 1 $\mathrm{cm}$ of water. Root samples were then scanned in grey scale at 600 dpi using a desktop scanner (Epson flatbed scanner) to record the phenotypic data on total root length $(\mathrm{RL} ; \mathrm{cm})$, total root surface area $\left(\mathrm{RSA}, \mathrm{cm}^{2}\right)$, root diameter $(\mathrm{RD}, \mathrm{mm})$ and total root volume $\left(\mathrm{RV} ; \mathrm{cm}^{3}\right)$, seminal root number (SRN) and lateral root number (LRN). The grey scale images obtained in tiff format were analysed using WinRHIZO Pro software (v2009, Regent Instruments, Montreal, QC, Canada). In addition, other root traits such as root hair abundance (RHA) and root dry weight (RDW; $\mathrm{mg}$ ) were recorded. RHA was recorded on the basis of scale: 1absent, 2- few, 3- medium, 4- high, 5- very high. RDW was recorded after drying the root samples in a hot air oven at $70^{\circ} \mathrm{C}$ for $72 \mathrm{hr}$. The experiment was conducted in randomized complete block design (RCBD) with two replicates per treatment within each experiment (unstressed control and drought stressed). Each paper roll containing ten seedlings constituted an experimental unit and each five healthy and homogenous seedlings per genotype made one replicate.

Statistical analyses: The relative value of root traits viz. relative $R L\left(R \_R L\right)$, relative $R S A\left(R \_R S A\right)$, relative $R V\left(R \_R V\right)$, relative $R D\left(R \_R D\right)$, relative SRN (R_SRN), relative LRN (R_LRN) and relative RDW (R_RDW) were calculated as the averages of the 
PEG treatment divided by that of the controls and used for variance, PCA and cluster analysis using SAS (SAS version 9.3, 2009). Cluster analysis was done using Ward's agglomerative hierarchical procedure based on squared Euclidean distance (Ward, 1963). Pearson correlation coefficients were computed at $\mathrm{P}_{0.05}$ and $\mathrm{P}_{0.01}$ and correlation matrices were generated using $\mathrm{R}$.

\section{Results and Discussion}

The studied barley genotypes showed wide ranges under optimum as well as stress conditions for most of the root traits (Table 1). The phenotypic means were higher and ranges were wider for root traits under optimum conditions than under drought stress. Under optimum conditions, the mean values for total RL per seedling ranged from 5.05 to $97.25 \mathrm{~cm}$ in IC533320 in comparison to check variety $\mathrm{BH} 902(48.69 \mathrm{~cm}), \mathrm{SRN}$ from 1.50 to $10, \mathrm{RDW}$ ranged from 0.20 to $12.75 \mathrm{mg}$ per seedling. Individual traits exhibited variable CV values. The lowest CV was found for RD (12.25\%) while highest was detected for LRN (96.97\%) under control conditions. The trait LRN was highly variable as it ranged from 0.30 lateral roots per seedling (EC578821, IC49597) to as high as 31.40 in genotypes EC578821, EC578523, and thus account for high CV recorded. Under stress conditions, RL ranged from 4.21 (EC578255) to $52.96 \mathrm{~cm}$ (IC393980) with a mean of $24.52 \mathrm{~cm}$ compared to rainfed varieties RD2660 and RD2624 used as check having total RL 31.55 and $27.98 \mathrm{~cm}$, respectively. Mean RDW was $3.52 \mathrm{mg}$ under PEG, while the range was 0.20 to $16.40 \mathrm{mg}$ per seedling. PEG induced osmotic stress led to decline of $47.18-54.53 \%$ in RL, RSA and SRV. RDW showed a decline of $28.73 \%$ under stress, however, LRN was stimulated due to stress imposition, which may be an adaptive strategy of plant to increase the water absorption under low water availability.

The wide range of all root morphological traits recorded under stress indicates genotype specific response to PEG stress. The trait RHA did not follow any specific pattern among the tested germplasm accessions. However, few tolerant accessions such as IC410036, EC328980, RD2794, NDB1445, UPB1008,
PRB502, VLB85, JB58 and Norboo showed an increase in the RHAunder stress compared to control, which may be an adaptive strategy to maximize water absorption, and thus available area for effective water absorption. The physiological significance of root hairs for substantially increasing water uptake and tolerance to drought has also been established earlier (Tanaka et al., 2014; Wasson et al., 2012). Under the influence of drought stress, plants alter their growth morphology which leads to increase in below ground biomass. Higher root length was observed in some accessions (IC393980, DWRB101) while others had higher root number (IC547560, IC590135 and IC335811) suggesting a different mechanism underlying drought tolerance. When moisture is a limiting factor, selection of genotypes with high proportion of root growth might be crucial as per Wilson (1988) and Gowda et al. (2011). Here, genotypes with high root length, surface area and with larger root volume may help to maintain cell turgor and to survive during drought period. Root traits such as root length density, root number, volume, root diameter, maximum root depth, total root length/surface area, root branching and root hair have been advocated for their functional significance in relation to water absorption and drought avoidance in rice by Gowda et al. (2011). The results presented here corroborates earlier drought tolerance studies in cereal crops by Robinson et al. (2016), Al-Karaki et al. (2008) and Abdel-Ghani et al. (2015) in barley, Neelam et al. (2018) in rice, Huang et al. (2017) in Eruca and Tomar et al. (2016) in wheat who reported probable role of root traits as a measure of drought susceptibility or tolerance.

Analysis of variance based on relative values of root traits indicated that at $15 \%$ PEG treatment, differences among the tested germplasm accessions were highly significant $\left(\mathrm{P}_{0.01}\right)$ with respect to $R \_R L, R \_R S A, R \_R V, R \_R D, R \_S R N, R \_L R N$ and R_RDW (Table 2). PEG-6000 at 15\% concentration significantly inhibited the R_RL, R_RSA, R_RV and R_RDW in studied accessions, however, R_LRN was stimulated. R_RD was although not much influenced by PEG treatment. Few germplasm accessions IC393980, EC578389, IC082719, EC492318 IC329556, IC335811 and wild accessions of $H$. marinum ssp. gussoneanum (EC123148, EC123150, EC578252, EC578256)

Table 1: Range, means, coefficient of variations (CV) and percentage of reduction for 220 barley genotypes under optimum and drought stress conditions for seven root traits

\begin{tabular}{|c|c|c|c|c|c|c|c|c|c|}
\hline \multirow[t]{2}{*}{ Traits } & \multicolumn{5}{|c|}{ Optimum conditions (no stress) } & \multicolumn{3}{|c|}{ Drought stress (15\% PEG) } & \multirow[b]{2}{*}{$\begin{array}{l}\% \text { of } \\
\text { reduction }\end{array}$} \\
\hline & Min & Max & Mean $\pm S . E$ & $\begin{array}{l}\text { CV } \\
(\%)\end{array}$ & Min & $\operatorname{Max}$ & Mean $\pm S . E$ & $\begin{array}{l}\text { CV } \\
(\%)\end{array}$ & \\
\hline $\mathrm{RL}$ & 5.05 & 97.25 & $50.30 \pm 0.95$ & 27.89 & 4.21 & 52.96 & $24.52 \pm 0.59$ & 35.87 & 47.18 \\
\hline RSA & 0.42 & 15.19 & $8.60 \pm 0.18$ & 31.23 & 0.52 & 7.35 & $3.78 \pm 0.08$ & 32.70 & 51.87 \\
\hline RV & 0.01 & 0.29 & $0.12 \pm 0.00$ & 37.52 & 0.01 & 0.09 & $0.05 \pm 0.00$ & 33.60 & 54.53 \\
\hline $\mathrm{RD}$ & 0.27 & 0.76 & $0.54 \pm 0.00$ & 12.25 & 0.22 & 0.66 & $0.50 \pm 0.00$ & 14.48 & 5.99 \\
\hline SRN & 1.50 & 10.00 & $5.48 \pm 0.07$ & 18.19 & 1.10 & 9.00 & $5.37 \pm 0.07$ & 19.45 & 1.40 \\
\hline LRN & 0.30 & 31.40 & $6.04 \pm 0.40$ & 96.97 & 0.40 & 27.25 & $5.14 \pm 0.28$ & 96.97 & -72.21 \\
\hline RDW & 0.20 & 12.75 & $5.45 \pm 0.14$ & 36.77 & 0.20 & 16.40 & $3.52 \pm 0.11$ & 47.01 & 28.73 \\
\hline
\end{tabular}

Abbreviations: RL, total root length (cm); RSA, total root surface area $\left(\mathrm{cm}^{2}\right)$; RV, root volume $\left(\mathrm{cm}^{3}\right) ; \mathrm{RD}$, root diameter (mm); SRN, seminal root number; $\mathrm{LRN}$, lateral root number and RDW, root dry weight $(\mathrm{mg})$ 
Table 2 : Variance analysis and coefficient of variations among different root traits of barley germplasm accessions evaluated for drought tolerance

\begin{tabular}{llllllll}
\hline & R_RL & R_RSA & R_RV & R_RD & R_SRN & R_LRN & R_RDW \\
\hline Mean Square & $0.11^{* *}$ & $0.09^{* *}$ & $0.10^{* *}$ & $0.04^{* *}$ & $0.04^{* *}$ & $17.69^{* *}$ & $0.35^{* *}$ \\
Mean & 0.53 & 0.50 & 0.47 & 0.94 & 0.99 & 1.91 & 0.72 \\
Coefficient of Variation & 19.0 & 21.34 & 22.71 & 8.67 & 12.93 & 62.21 & 59.71 \\
SE & 0.10 & 0.10 & 0.10 & 0.08 & 0.13 & 1.19 & 0.43 \\
LSD at 5\% & 0.20 & 0.20 & 0.21 & 0.16 & 0.25 & 2.34 & 0.84 \\
\hline
\end{tabular}

The relative values of root traits were used for analysis of variance (For description, see the material and methods section). ${ }^{*}$ significant at $P_{0.01}$ level. Abbreviations: $R \_R L$, relative root length; $R \_R S A$, relative root surface area; $R \_R V$, relative root volume; $R \_R D$, relative root diameter; $R \_S R N$, relative seminal root number; $R \_L R N$, relative lateral root number and $R \_R D W$, relative root dry weight

maintained almost same or even higher root growth under PEG treatment in comparison to control, and thus proved to be highly drought tolerant. Earlier variability in drought tolerance, based on root traits, have also been well documented in maize (Tuberosa et al., 2002), triticale (Grzesiak et al., 2003), wheat (Manschadi et al., 2006), rice (Henry et al., 2012; Uga et al., 2013) and lentil (Sarker et al., 2005). In many studies, grain yield and plant growth were found to be positively associated with a deep and extended root system in cereals (Pandey et al., 2000a, b; Chloupek et al., 2013). Thus, root architecture traits studied in the present work could potentially be considered as selection criteria to improve plant growth under drought and hence yield.

The matrix developed for correlations among different root traits along with correlation coefficients indicated that R_RL,
R_RSA, R_RV and R_RDW were positively and significantly correlated with each other at P0.01 (Fig. 1). R_RD was positively correlated with R_SRN and negatively correlated with $R \_R L$ and $R \_L R N\left(P_{0.01}\right)$. R_SRN was positively correlated with all the traits except $R \_R L$ and $R \_L R N$, while $R \_L R N$ was found to be not correlated with any of the root traits mentioned above, except weak negative association with $\mathrm{R} \_\mathrm{RD}\left(-0.21, \mathrm{P}_{0.01}\right)$. Thus, focusing on the traits such as RL, RSA and RDW would provide sufficient information to evaluate genetic diversity for seedling root architecture in barley germplasm for massive screening in a short period of time. Other studies have shown positive correlations between seedling root attributes, adult root characteristics and grain yield (Nass and Zuber, 1971; Chloupek et al., 2013) which might indicate the importance of early seedling root traits as a predictor for plant growth and grain yield. The
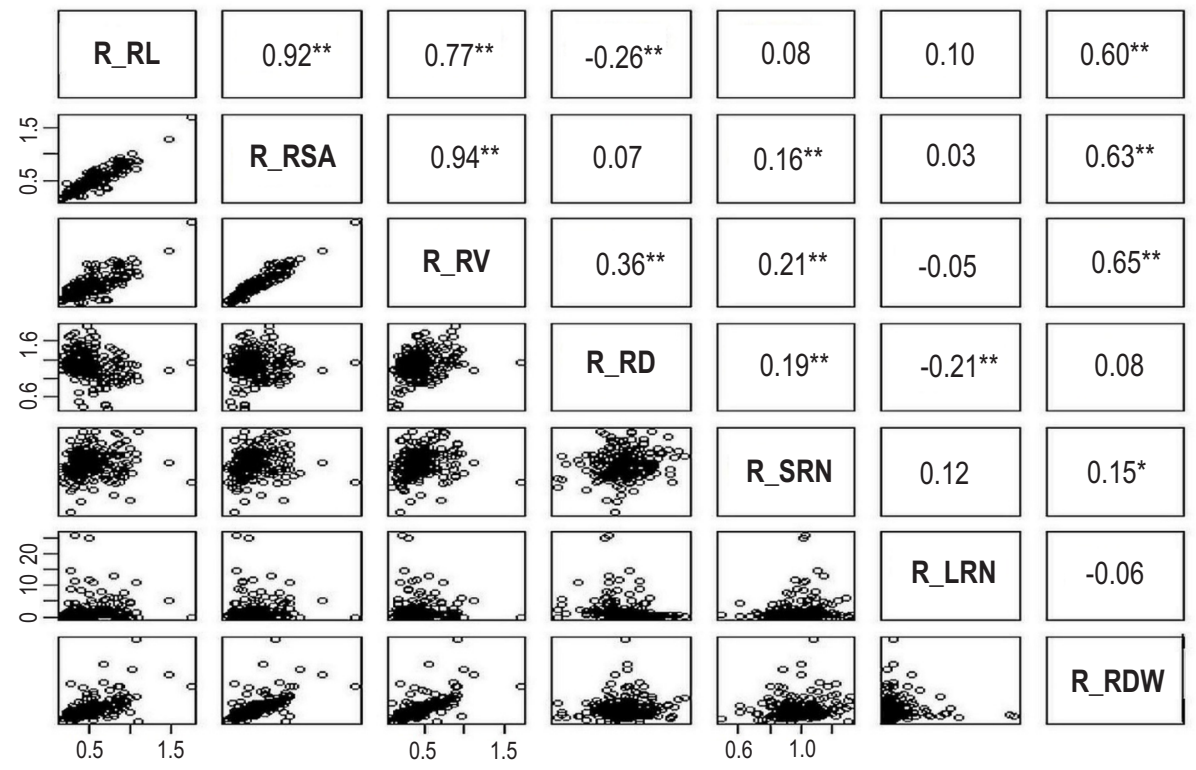

Fig. 1: The pairwise correlations among different root traits of barley germplasm accessions evaluated for drought tolerance. The relative value of root traits were used for correlation analysis (For description, see the material and methods section). The upper-right panels show the numerical values of the correlations (rounded to two decimal places). The ${ }^{*}$ and ${ }^{* *}$ indicate correlation significance at $P_{0.05}$ and $P_{0.01}$ levels of probability, respectively. The lower-left panels show the scatter plots. Abbreviations: $R \_R L$, relative root length; $R \_R S A$, relative root surface area; $R \_R V$, relative root volume; $R \_R D$, relative root diameter; $R \_S R N$, relative seminal root number; $R \_L R N$, relative lateral root number and $R \_R D W$, relative root dry weight. 


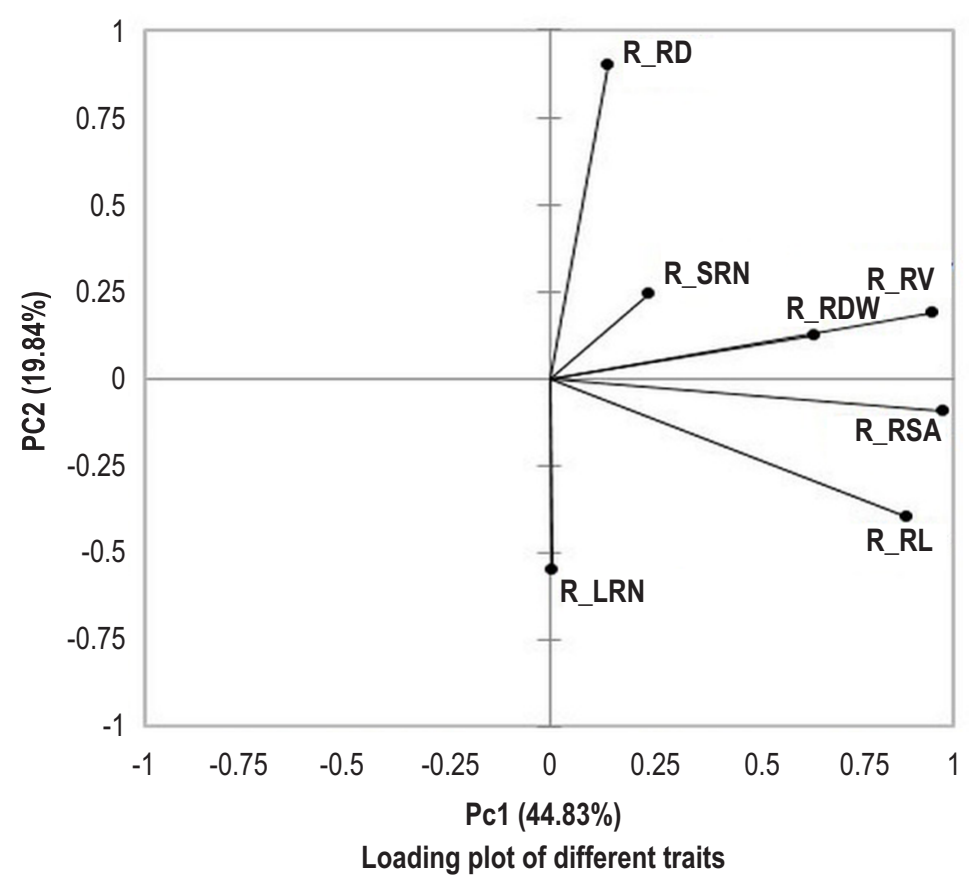

Fig. 2 : Biplot of different variables loaded on PC1 and PC2. The relative value of root traits were used for biplot (For description, see the material and methods section). Abbreviations: $R \_R L$, relative root length; $R \_R S A$, relative root surface area; $R \_R V$, relative root volume; $R \_R D$, relative root diameter; R_SRN, relative seminal root number; $R \_L R N$, relative lateral root number and R_RDW, relative root dry weight.

cluster analysis carried out by Ward's agglomerative hierarchical method categorized 220 barley accessions into five major clusters. Based on low PC1 scores, the first cluster was drought susceptible and 56 accessions grouped under this cluster. Cluster II was constituted by drought tolerant genotypes (32 accessions). Cluster III being slightly-medium drought tolerant (moderate PC scores) grouped 42 accessions including wild species $H$. vulgare ssp. spontaneum and $H$. murinum ssp. leporinum. Cluster IV accessions showed highest PC1 and low PC2 scores and was constituted by eightmost drought tolerant accessions including 3 accessions of wild barley $H$. marinum ssp. gussoneanum. Highly drought sensitive accessions with low PC1 scores grouped under cluster $\mathrm{V}$, which was the largest cluster with 82 accessions. The maximum differences were observed between accessions under cluster $\mathrm{V}$ and IV indicating genetic distance among them due to different genetic constitutions. Hence, divergent parents selected from these clusters can be utilized in breeding program for barley improvement under rain-fed conditions, which is the main breeding target in India. Present results are in congruence with Tomar et al. (2016) and Huang et al. (2017), who also categorized genotypes into highly tolerant, sensitive, moderately sensitive and tolerant while studying molecular and agro-morphological characterisation of root architecture for drought tolerance in wheat and Eruca, respectively.

PCA is designed to transform the original data into new uncorrelated variables known as principal components which are useful for identification of determinants of quantitative trait variability when a large number of accession are studied (Alvin,
2002). PCA based on relative value of seven root traits as drought tolerance indices showed that the first three components with latent root values more than 1 accounted for $80.50 \%$ of the total variation (Table 3). First principal component (PC1) accounted for $44.83 \%$ of the total multivariate variation, PC2 accounted for

Table 3 : Principal Component (PC) with latent root values more than 1 in barley germplasm accessions evaluated for drought tolerance

\begin{tabular}{llll}
\hline Eigen value & 3.13 & 1.39 & 1.11 \\
Proportional variation (\%) & 44.83 & 19.84 & 15.87 \\
Cumulative variation (\%) & 44.83 & 64.68 & 80.50 \\
Traits & PC1 & PC2 & PC3 \\
R_RL & 0.49 & -0.34 & -0.12 \\
R_RSA & 0.55 & -0.08 & -0.06 \\
R_RV & 0.54 & 0.16 & -0.02 \\
R_RD & 0.08 & 0.77 & 0.14 \\
R_SRN & 0.15 & 0.21 & 0.75 \\
R_LRN & 0.01 & -0.46 & 0.63 \\
R_RDW & 0.36 & 0.11 & -0.06 \\
\hline
\end{tabular}

The relative values of root traits were used for Principal Component Analysis (For description, see the material and methods section). Bold values indicate the highest values among all three PCs for each trait which represents relative contribution of respective $\mathrm{PC}$ in explaining the variation. Abbreviations: $R \_R L$, relative root length; $R \_R S A$, relative root surface area; $R \quad R V$, relative root volume; $R \quad R D$, relative root diameter; R_SRN, relative seminal root number; R_LRN, relative lateral root number and $R \_R D W$, relative root dry weight 
$19.84 \%$ and PC3 for $15.87 \%$ of the variation. Plot of first two Eigen vectors for 220 barley genotypes was performed to detect any possible discriminations among barley genotypes based on row morphology, origin of samples or place of collection and genetic background (Fig. 2). The biplot indicated that PC1 had high contributions through traits R_RSA, R_RV, R_RL and R_RDW. $R \_R D$ had the highest contribution in PC2 while R_LRN showed negative contribution. R_SRN and R_LRN were responsible for highest contribution to the total variation in PC3. The traits RL, RSA and RDW accounted for highest variation in germplasm. In this study, the distribution of germplasm within PC plots and clusters had no apparent relationship with the row-type (two-row versus six-rowed type), geographical origin or biological status (landrace, improved cultivar or breeding line etc.). However, PCA, biplot loading and cluster analysis give comprehensive information of traits and accessions, which are critically contributing towards drought tolerance. Accessions IC393980, IC082719, IC329556, EC492318, EC578789, EC578790, IC335811 and wild barley H. marinum ssp. gussoneanum proved to be the most drought tolerant based on high PC scores. Earlier, PCA has been successfully used for studying drought tolerance in soybean (Kron et al., 2008), tomato (Shamimi et al., 2014), wheat (Peleg et al., 2005) and Eruca (Huang et al., 2017).

In conclusion, substantial variation was detected among studied barley genotypes in their response to PEGinduced osmotic stress for most of the traits recorded. The outstanding genotypes with high PC scores such as IC082719, IC329556, EC492318, EC578789, EC578790, IC335811 may prove to be valuable for genetic improvement not only in barley itself but also in other cereal crops as they are drought tolerant lines from a drought tolerant species. Present study entrusted germination paper roll method to induce PEG-simulated drought stress due to several advantages such as easy scoring, increased repeatability, high heritability and precise highthroughput phenotyping compared with field/pot screening. However, it is obvious that the artificial screening conditions employed in this study may not properly reflect field conditions, and thus require validation through field evaluation. In this regard, a panel of genotypes with contrasting response to drought stress (most tolerant versus most susceptible) identified in the present study is being validated under drought stress conditions in field and candidate gene association study to identify genomic regions associated with drought tolerance will be attempted in the next phase of this study.

\section{Acknowledgments}

Authors acknowledge use of research facilities available at ICAR-NBPGR. The use of Root scanner and WinRhizo Pro software available at Division of Plant Physiology, ICAR-IARI, New Delhi is also duly acknowledged and authors express sincere thanks to Dr. V. Chinnusamy, Principal Scientist / Head and Dr. Rakesh Pandey, Principal Scientist, Division of Plant Physiology, ICAR-IARI, Pusa Campus, New Delhi, India.

\section{References}

Abdel-Ghani, A.H., K. Neumann, C. Wabila, R. Sharma, S. Dhanagond, J. SaedOwais, A. Börner, A. Graner and K. Benjamin: Diversity of germination and seedling traits in a spring barley (Hordeum vulgare L.) collection under drought simulated conditions. Genet. Resour. Crop Evol., 62, 275-292 (2015).

Al-Karaki, G.N.: Response of wheat and barley during germination to seed osmopriming at different water potential. J. Agron. Crop Sci., 181, 229-235(2008)

Alvin, C.R.: Methods of Multivariate Analysis. $3^{\text {rd }}$ Edn., John Wiley \& Sons, Inc., New York, USA (2002).

Ashraf, M., M. Ozturk and H.R. Athar: Salinity and water stress: Improving crop efficiency 2009 Ed., Springer-Verlag, Inc., Berlin, Germany (2009).

Ceccarelli, S., S. Grando, V. Shevstov, H. Vivar, A. Yahayaoui, M. Elbhoussini and M. Baum: The ICARDA strategy for global barley improvement. Barley and Wheat Newsletter, 18, 3-12 (1999).

Chen, G.X., T. Krugman, T. Fahima, K.G. Chen, Y.G. Hu, M. Roder, E. Nevo and A. Korol: Chromosomal regions controlling seedling drought resistance in Israeli wild barley, Hordeum spontaneum C Koch. Genet. Resour. Crop Evol., 57, 85-99 (2010).

Chloupek, O., V. Dostal, T. Streda, V. Psota and O. Dvorackova: Drought tolerance of barley varieties in relation to their root system size. Plant Breed., 129, 630-636 (2013).

Christopher, J., M. Christopher, R. Jennings, S. Jones, S. Fletcher and A. Borrell: QTL for root angle and number in a population developed from bread wheats (Triticum aestivum) with contrasting adaptation to water-limited environments. Theor. Appl. Genet., 126, 1563$1574(2013)$.

Diab, A.A., B. Teulat-Merah, D. This, N.Z. Ozturk, D. Benscher and M.E. Sorrells: Identification of drought-inducible genes and differentially expressed sequence tags in barley. Theor. Appl. Genet., 109, 1417-1425 (2004).

Druka, A., K. Sato and G.J. Muehlbauer: Genome Analysis: The State of Knowledge of Barley Genes. In: Production, Improvement, and Usesof Barley (Eds.: S.E. Ullrich). Blackwell Publishing Ltd., United Kingdom, pp. 1-13 (2011).

FAO: Food and Agricultural Commodities Production. http:// faostat. fao. org/ site/339/default.aspx (2017).

Gowda, V.R.P., A. Henry, A. Yamauchi, H.E. Shashidhar and R. Serraj: Root biology and genetic improvement for drought avoidance in rice. Field Crop Res., 122,1-13 (2011).

Grzesiak, S., M.T. Grzesiak, W. Filek and J. Stabryta: Evaluation of physiological screening tests for breeding drought resistant triticale (Triticosecale Wittmack). Acta Physiologiae Plantarum, 25, 29-37 (2003).

Henry, A., A.J. Cal, T.C. Batoto, R.O. Torres and R. Serraj: Root attributes affecting water uptake of rice (Oryza sativa) under drought. J. Exp. Bot., 63,4751-4763 (2012).

Hoagland, D.R. and D.I. Arnon: The Water-culture method for growing plants without soil. California Agricultural Experiment Station, Circular, 347, 1-132 (1950).

Huang, B., J. Su, G. Zhang, X. Luo, H. Wang, Y. Gao, G. Ma, J. Wang, D. Cai, X. Zhang and B. Huang: Screening for Eruca genotypes tolerant to polyethylene glycol-simulated drought stress based on the principal component and cluster analyses of seed germination and early seedling growth. Plant Genet. Resour., 15, 187-193 (2017).

Kaur, V., R. Yadav and D.P. Wankhede: Linseed genetic resources for climate change intervention and future breeding. J. Appl. Nat. Sci., 9, 1112-1118 (2017).

Kaur, V., S. Kumar, R. Yadav, D.P. Wankhede, J. Aravind, J. Radhamani, 
J.C. Rana and A. Kumar: Analysis of genetic diversity in Indian and exotic linseed germplasm and identification of trait specific superior accessions. J. Environ. Biol., 39, $702-709$ (2018).

Kaur, V., S. Singh and R.K. Behl: Heat and drought tolerance in wheat Integration of physiological and genetic platforms for better performance under stress. Ekin J., 2, 1-14 (2016).

Kron, A.P., G.M. Souza and R.V. Ribeiro: Water deficiency at different developmental stages of Glycine max can improve drought tolerance. Bragantia, 67, 43-49 (2008).

Kumar, G.R., K. Sakthivel, R.M. Sundaram, C.N. Neeraja, S.M. Balachandran, N.S. Rani, B.C. Viraktamath and M.S. Madhav: Allele mining in crops: Prospects and potentials. Biotech. Adv., 28 451-61 (2010).

Kumar, V., R. Kumar, R.P.S. Verma, A. Verma and I. Sharma: Recent trends in breeder seed production of barley (Hordeum vulgare) in India. Indian J. Agric. Sci., 83, 576-578 (2013).

Manschadi, A.M., J. Christopher, P. deVoil and G.L. Hammer: The role of root architectural traits in adaptation of wheat to water-limited environments. Funct. Plant Biol., 33, 823-837 (2006).

Nass, H.G. and M.S. Zuber: Correlation of corn (Zea mays L.) roots toearly in development to mature root development. Crop Sci., 11 655-658 (1971).

Neelam, K., G. Sahi, K. Kumar and K. Singh: Identification of drought stress tolerance in wild species germplasm of rice based on lea and root morphology. Plant Genet. Resour., 16, 289-295 (2018).

Pandey, H.C., M.J. Baig, A. Chandra and R.K. Bhatt: Drought stress induced changes in lipid peroxidation and antioxidant system in genus Avena. J. Environ. Biol., 31, 435-440 (2010).

Pandey, R.K., J.W. Maranville and M.M. Chetima: Deficit irrigation and nitrogen effects on maize in a Sahelian environment II. Shoot growth, nitrogen uptake and water extraction. Agric. Water Manage., 46, 15-27 (2000a).

Pandey. R.K., J.W. Maranville and A. Admou: Deficit irrigation and nitrogen effects on maize in a Sahelian envrionment. I. Grain yield and yield components. Agric. Water Manage., 46, 1-13 (2000b).

Peleg, Z., T. Fahima, S. Abbo, T. Krugman, E. Nevo, D. Yakir and Y Saranga: Genetic diversity for drought resistance in wild emmer wheat and its eco-geographical associations. Plant Cell Environ. 28, 176-191 (2005)

Pennisi, E.: Plant genetics: The blue revolution, drop by drop, gene by gene. Science, 320, 171-173 (2008)

Robinson, H., L. Hickey, C. Richard, E. Mace, A. Kelly, A. Borrell, J. Franckowiak and G. Fox: Genomic regions influencing seminal root traits in barley. The Plant Genome, 9, 1-13 (2016).

Sarker, A., W. Erskine and M. Singh: Variation in shoot and root characteristics and their association with drought tolerance in lentil landraces. Genet. Resour. Crop Evol., 52, 87-95 (2005).

SAS Institute: Statistical analysis software system, Version 9.3. SAS Institute, Cary, NC, USA(2009).
Shamimi, F., S.M. Saqlan, H.U.R. Athar and A. Waheed: Screening and selection of tomato genotypes for drought tolerance using multivariate analysis. Pak J. Bot., 46, 1165-1178 (2014).

Shavrukov, Y., N.K. Gupta, J. Miyazaki, M.N. Baho, K.J. Chalmers and M. Tester: HvNax3-a locus controlling shoot sodium exclusion derived from wild barley (Hordeum vulgare ssp. spontaneum). Funct. Integr. Genomics, 10, 277-291 (2010).

Tanaka, N., M. Kato, R. Tomioka, R. Kurata, Y. Fukao, T. Aoyama and M. Maeshima: Characteristics of a root hair-less line of Arabidopsis thaliana under physiological stresses. J. Exp. Bot., 65, 1497-1512 (2014).

Teulat, B., C. Borries and D. This: New QTLs identified for plant water status, water-soluble carbohydrate and osmotic adjustment in a barley population grown in a growth-chamber under two water regimes. Theor. Appl. Genet., 103, 161-170 (2001).

Teulat, B., N. Zoumarou-Wallis, B. Rotter, B. Salem, H. Bahri and D. This: QTL for relative water content in field-grown barley and their stability across Mediterranean environments. Theor. Appl. Genet., 108, 181-188 (2003)

Tomar, R.S.S., S. Tiwari, Vinod, B.K. Naik, S. Chand and R. Deshmukh: Molecular and morpho-agronomical characterization of root architecture at seedling and reproductive stages for drought tolerance in wheat. PLOSONE, 11, e0156528 (2016).

Tuberosa, R., M.C. Sanguineti, P. Landi, M.M. Giuliani, S. Salvi and S. Conti: Identification of QTLs for root characteristics in maize grown in hydroponics and analysis of their overlap with QTLs for grain yield in the field at two water regimes. Plant Mol. Biol., 48, 697-712 (2002).

Uçarlı, C., L.J. McGuffin, S. Çaputlu, A. Aravena and F. Gürel: Genetic diversity at the Dhn3 locus in Turkish Hordeum spontaneum populations with comparative structural analyses. Sci. Rep., 6 , 20966 (2016).

Uga, Y., K. Sugimoto, S. Ogawa, J. Rane, M. Ishitani, N. Hara, Y. Kitomi, Y, Inukai, K. Ono, N. Kanno, H. Inoue, H. Takehisa, R. Motoyama, Y. Nagamura, J. Wu, T. Matsumoto, T. Takai, K. Okuno and M. Yano: Control of root system architecture by DEEPER ROOTING 1 increases rice yield under drought conditions. Nat. Genet., 45, 1097-1102 (2013).

Ward, J.H.: Hierarchical grouping to optimize an objective function. J. Am. Stat. Assoc., 58, 236-244 (1963).

Wasson, A.P., R.A. Richards, R. Chatrath, S.C. Misra, S.V. Prasad, G.J. Rebetzke, J.A. Kirkegaard, J. Christopher and M. Watt: Traits and selection strategies to improve root systems and water uptake in water-limited wheat crops. J. Exp. Bot., 63, 3485-3498 (2012).

Wilson, J.B.: A review of evidence on the control of shoot: Root ratio, in relation to models. Ann. Bot., 61, 433-449 (1988).

Zhu, J., S.M. Kaeppler and J.P. Lynch: Mapping of QTLs for lateral root branching and length in maize (Zea mays L.) under differential phosphorus supply. Theor. Appl. Genet., 111, 688-695(2005). 